\title{
Plasmalemma vesicle-associated protein: A crucial component of vascular homeostasis (Review)
}

\author{
LING GUO $^{1 *}$, HONGYAN ZHANG ${ }^{2 *}$, YINGLONG HOU ${ }^{3}$, TIANSHU WEI ${ }^{4}$ and $\mathrm{JU}_{\text {LIU }}^{1}$ \\ ${ }^{1}$ Laboratory of Microvascular Medicine, Medical Research Center, Shandong Provincial Qianfoshan Hospital, \\ Shandong University, Jinan, Shandong 250014; ${ }^{2}$ Department of Cardiovascular Medicine, \\ Xintai City People's Hospital Affiliated to Taishan Medical University, Xintai, Shandong 271200; \\ ${ }^{3}$ Department of Cardiology, Shandong Provincial Qianfoshan Hospital, Shandong University, Jinan, \\ Shandong 250014, P.R. China; ${ }^{4}$ Centre for Epidemiology and Biostatistics, \\ School of Population and Global Health, The University of Melbourne, Victoria 3010, Australia
}

Received February 26, 2016; Accepted June 27, 2016

DOI: $10.3892 /$ etm.2016.3557

\begin{abstract}
Endothelial subcellular structures, including caveolae, fenestrae and transendothelial channels, are crucial for regulating microvascular function. Plasmalemma vesicle-associated protein (PLVAP) is an endothelial cell-specific protein that forms the stomatal and fenestral diaphragms of blood vessels and regulates basal permeability, leukocyte migration and angiogenesis. Loss of PLVAP in mice leads to premature mortality due to disrupted homeostasis. Evidence from previous studies suggested that PLVAP is involved in cancer,
\end{abstract}

Correspondence to: $\mathrm{Dr} \mathrm{Ju}$ Liu, Laboratory of Microvascular Medicine, Medical Research Center, Shandong Provincial Qianfoshan Hospital, Shandong University, 16766 Jingshi Road, Jinan, Shandong 250014, P.R. China

E-mail: ju.liu@sdu.edu.cn

Dr Yinglong Hou, Department of Cardiology, Shandong Provincial Qianfoshan Hospital, Shandong University, 16766 Jingshi Road, Jinan, Shandong 250014, P.R. China

E-mail: houyinglong2013@hotmail.com

*Contributed equally

Abbreviations: BRB, blood-retinal barrier; BRECs, bovine retinal endothelial cells; DR, diabetic retinopathy; ECs, endothelial cells; FD, fenestral diaphragms; HCC, hepatocellular carcinoma; HUVECs, human umbilical vein endothelial cells; LECs, lymphatic endothelial cells; NDP, Norrie disease pseudoglioma; PLVAP, plasmalemma vesicle-associated protein-1; PLNs, peripheral lymph nodes; PI3K, phosphatidylinositol 3-kinase; p38MAPK, p38 mitogen-activated protein kinase; PMA, phorbol myristate acetate; SCI, spinal cord injury; SD, stomatal diaphragm; TECs, transendothelial channels; TG, transplant glomerulopathy; VEGF, vascular endothelial growth factor; VVOs, vesiculo-vacuolar organelles; ZO-1, zonula occludens-1

Key words: plasmalemma vesicle-associated protein-1, endothelial cell, diaphragms, permeability, homeostasis traumatic spinal cord injury, acute ischemic brain disease, transplant glomerulopathy, Norrie disease and diabetic retinopathy. Specifically, PLVAP expression has been demonstrated to be upregulated in these diseases, accompanied by pro-angiogenic or pro-inflammatory responses. Therefore, PLVAP is considered a novel therapeutic target, in addition to an endothelial cell marker. The present review summarizes the structure and functions of PLVAP, and its roles in pathophysiological processes.

\section{Contents}

1. Introduction

2. PLVAP protein structure

3. PLVAP protein expression pattern

4. Regulation of PLVAP expression

5. Roles of PLVAP in physiological processes

6. PLVAP and diseases

7. Conclusion

\section{Introduction}

The vascular endothelium, which lines the inner surface of the entire cardiovascular system $(1,2)$, performs vital functions in maintaining permeability, vessel wall integrity and homeostasis, and preventing thrombosis (1-4). Caveolae, transendothelial channels (TECs), and fenestrae are subcellular organelles that are present in a subset of endothelial cells (ECs) that regulate microvascular permeability (1-3). Caveolae are plasma membrane invaginations displaying a thin stomatal diaphragm (SD) in their necks $(5,6)$. Fenestrae, which are 60-80 nm transcellular pores spanned by fenestral diaphragms (FDs), consist of radial fibrils on their lumenal side (7-9). TECs are spanned by two stomatal diaphragms, and arise interspersed with fenestrae in discontinued areas of ECs (9-11).

Plasmalemma vesicle-associated protein (PLVAP) is commonly considered to be endothelium-specific as it is an antigen for two classic antibodies of the endothelium: Mouse 
endothelial cell antigen (MECA)-32 and pathologische anatomie Leiden-endothelium (PAL-E) $(4,10,12,13)$. PLVAP was the first molecular component of fenestrae to be identified, and is essential for the development of FDs and SDs in ECs $(14,15)$. Previous studies have reported roles for PLVAP in the regulation of basal permeability, leukocyte migration and angiogenesis (16-20). In addition, upregulation of PLVAP has previously been associated with cancer, traumatic spinal cord injury, transplant glomerulopathy (TG), ischemic brain disease and ocular disease $(16,17,21-26)$ (Table I). Furthermore, PLVAP has been investigated as a novel target in cancer therapy (27). The present review provides a detailed description of the current understanding of the biological properties and functions of PLVAP.

\section{PLVAP protein structure}

PLVAP, a type II integral membrane glycoprotein with a molecular weight of $\sim 60 \mathrm{kDa}$, forms dimers in situ and binds to heparin at physiological $\mathrm{pH}(3,5,28,29)$. PLVAP consists of three sections: A short (27-amino acid) intracellular tail, a transmembrane domain and a long (358-amino acid) extracellular C-terminal domain (6,30) (Fig. 1). The intracellular domain of PLVAP consists of two short identical stretches of amino acids: One is adjacent to the transmembrane region (8 amino acids) and contains a putative caveolin-1 binding domain, whereas the other is at the extreme $\mathrm{N}$-terminus ( 7 amino acids) of the protein (6). The extracellular domain consists of four $\mathrm{N}$-glycosylation sites, a proline-rich region near the $\mathrm{C}$-terminus and two large coiled-coil domains (31) (Fig. 1). Every seventh amino acid of the $\alpha$-helix of the coiled-coil domain is hydrophobic to facilitate the formation of an intermolecular superhelix (4).

\section{PLVAP protein expression pattern}

The PLVAP protein is restricted to the membrane of a subset of ECs in the normal microvasculature (3). The highest levels of PLVAP were detected in the lungs, kidneys, spleen, endocrine glands and digestive tract (28). Notably, PLVAP is not expressed in the ECs of large vessels, with the exception of the endocardial lining of the heart chambers $(6,32)$.

\section{Regulation of PLVAP}

Vascular endothelial growth factor (VEGF), which stimulates increased vascular permeability and angiogenesis, is the primary regulator of PLVAP (33). However, the reports of the effects of VEGF on PLVAP expression have been conflicting. Hofman et al (34) suggested that PLVAP was directly or indirectly induced by VEGF, as VEGF and PLVAP (the then PAL-E) were revealed to simultaneously be present on the retina of diabetic patients with retinal vascular leakage. Consistent with this, Strickland et al (33) demonstrated that treatment of human umbilical vein ECs (HUVECs) with VEGF increased the mRNA and protein expression levels of PLVAP via activation of the VEGF receptor 2 (33). Furthermore, this effect was attenuated by an anti-VEGF monoclonal antibody, and was reported to be mediated via the phosphatidylinositol 3-kinase (PI3K) and p38 mitogen-activated protein kinase (p38MAPK) signaling pathways (33) (Fig. 2). In addition,

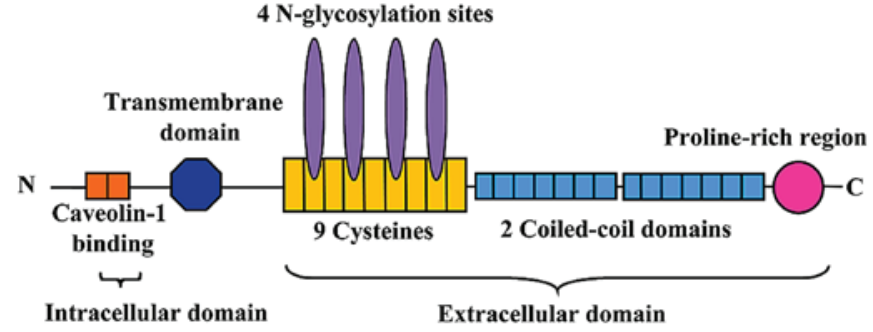

Figure 1. Protein structure of plasmalemma vesicle-associated protein.

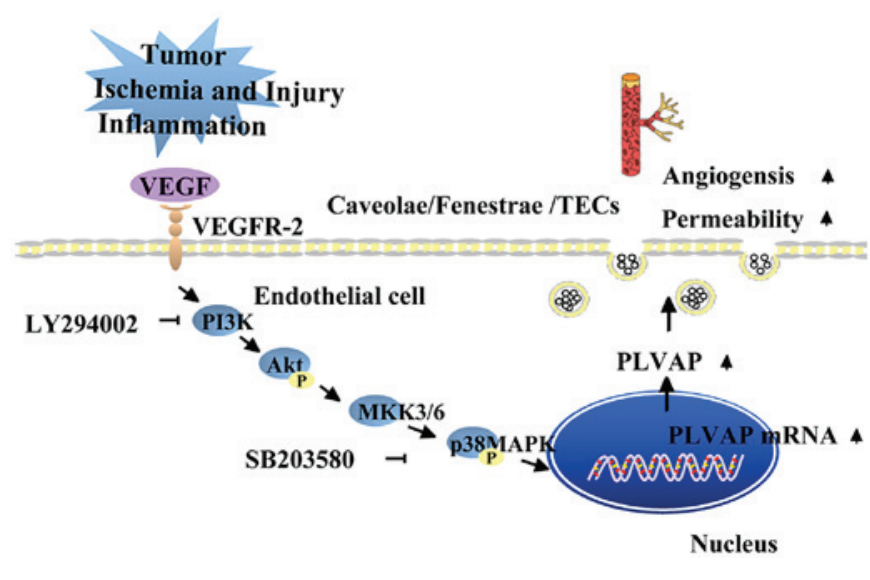

Figure 2. Regulation of PLVAP expression. VEGF signaling stimulates the expression of PLVAP via activation of the PI3K and p38MAPK signaling pathways. The PI3K inhibitor LY294002 and p38MAPK inhibitor SB203580 decrease the mRNA and protein expression levels of PLVAP. PLVAP, plasmalemma vesicle-associated protein; VEGF, vascular endothelial growth factor; PI3K, phosphatidylinositol 3-kinase; p38MAPK, p38 mitogen-activated protein kinase; VEGFR-2, vascular endothelial growth factor receptor-2; TECs, transendothelial channels; MKK3/6, mitogen-activated protein kinase kinase $3 / 6$

treatment with the PI3K inhibitor LY294002 or the p38MAPK inhibitor SB203580 induced a dose-dependent decrease in the mRNA and protein expression levels of PLVAP (33). However, experiments using caveolin-1-null mice suggested that PLVAP expression in the lungs was negatively regulated by VEGF (35). Notably, the PLVAP expression level remained unchanged in caveolin-2-null mice under identical experimental conditions (35). These seemingly contradictory results suggested that other endothelial proteins, such as caveolin-1, may affect VEGF-mediated regulation of PLVAP expression. In addition, the effects of increased VEGF expression on PLVAP expression may vary across different organs and/or species $(33,35)$. PLVAP expression has also been shown to be regulated by phorbol myristate acetate (PMA), an activator of protein kinase C (14). The treatment of EC cultures with PMA resulted in the upregulation of PLVAP expression in a dose-dependent and time-dependent manner (14). Furthermore, PMA-induced upregulation of PLVAP expression was hypothesized to be dependent on the activation of the extracellular signal-regulated protein kinase 1/2-MAPK signaling pathway (14).

\section{Roles of PLVAP in physiological processes}

PLVAP forms SDs and FDs. PLVAP, which is the only known molecular component of SDs and FDs $(14,28)$, forms 
Table I. Functions of plasmalemma vesicle-associated protein in diseases.

\begin{tabular}{llc}
\hline Disease & \multicolumn{1}{c}{ Function } & Refs. \\
\hline Cancer & Angiogenesis $\uparrow$ & $(16-18,43)$ \\
& Permeability $\uparrow$ & $(32,43)$ \\
Traumatic spinal cord injury & Angiogenesis $\uparrow$ & $(25)$ \\
Transplant glomerulopathy & Permeability $\uparrow$ & $(26,47)$ \\
Acute ischemic brain disease & Permeability $\uparrow$, angiogenesis $\uparrow$ & $(21)$ \\
Norrie disease & Permeability $\uparrow$, angiogenesis $\uparrow$ & $(22-24)$ \\
Diabetic retinopathy & Permeability $\uparrow$, angiogenesis $\uparrow$ & \\
\hline
\end{tabular}

Arrows indicate upregulation.

homodimers that are cross-linked in situ $(5,14,28)$. The upregulation of PLVAP expression upon treatment of EC cultures with PMA was associated with the de novo formation of SDs and FDs that were demonstrated to contain PLVAP (14). In addition, silencing of PLVAP mRNA expression inhibited de novo diaphragm formation in caveolae, TECs and fenestrate $(14,15)$, and knockout of the PLVAP gene in mice resulted in the complete absence of SDs and FDs (10). These findings suggested that PLVAP is required for the formation of SDs and FDs in ECs.

It has been hypothesized that PLVAP is responsible for the formation of the radial fibrils that constitute both SDs and FDs (31). To elaborate, these diaphragms consist of thin fibrils originating at the inner surfaces that then intertwine into a knot at the lumenal center of caveolae, fenestrae or TECs (4). At present, it is unclear whether PLVAP is the only protein present in the diaphragms (31). Various functional groups at the C-terminus of PLVAP potentially offer binding sites for other PLVAPs, causing the fibrils to interweave in the observed manner (31). In addition, the polysaccharides occupying $\sim 15 \%$ of the molecular weight of PLVAP near the membrane may support the membrane and maintain the fibrils in their correct position (4). Furthermore, interactions between PLVAP C-termini and the existence of a rigid structure attaching PLVAP to the cytoskeleton may contribute to the stabilization of the diaphragms (4).

PLVAP regulates basal permeability and maintains the integrity of blood vessels. PLVAP is restricted to a subset of capillary endothelial linings, as demonstrated by immunofluorescence using anti-PLVAP antibodies $(5,28)$. On a C57BL/6N genetic background, PLVAP-null embryos died before birth and suffered from edema, hemorrhages, and defects to the vascular wall of subcutaneous capillaries (36). Furthermore, diaphragms were missing from caveolae of the subcutaneous capillaries and endocardium ECs of PLVAP-null mice (36). In addition, capillaries of the pancreas and kidney peritubular interstitium of PLVAP-null mice demonstrated a complete absence of diaphragms in fenestrae, caveolae, and TECs (36). Transmission electron microscopy confirmed the lack of endothelial integrity, detecting extensive defects in the endothelial lining of capillaries (36).

Similarly, Stan et al (10) demonstrated that the deletion of the PLVAP gene in mice of a mixed BALB/c-C57Bl/6J-129Sv/J background resulted in decreased survival due to the absence of diaphragms. In particular, the loss of diaphragms disrupted the barrier function of fenestrated capillaries, causing a major leak of plasma proteins, and leading to hypoproteinemia, hypertriglyceridemia, and an increased plasma concentration of chylomicron remnants (10). As a result, PLVAP-null mice suffered from premature death due to severe enteropathy and edema of the intestine, kidneys, and pancreas (10). In addition, the results of an Evans Blue dye extravasation assay indicated that the intestinal capillaries displayed the highest rate of protein leakage (10). Conversely, endothelial reconstitution of PLVAP by breeding with PLVAP transgenic mice rescued the PLVAP-null phenotype and restored the diaphragms of fenestrae and caveolae (10). As human influenza hemagglutinin (HA)-tagging of PLVAP did not alter its behavior in cultured cells, VEC-PVIHA-HA transgenic mice were generated that expressed PV1-HA under the control of the vascular endothelial-cadherin promoter and a 5 '-intronic enhancer element (10,37). These mice demonstrated $\sim 30-50 \%$ reconstitution of PLVAP expression, in addition to the restoration of FDs/SDs in the lungs, adrenal glands, kidneys, pancreas, thyroid and intestine, which led to improved survival (10). Therefore, PLVAP is crucial for the regulation of vascular permeability (Fig. 3).

PLVAP facilitates leukocyte trafficking. The migration of leukocytes from the bloodstream into tissues via a transcellular pathway through the endothelium is one of the central paradigms of inflammation and immunity $(19,38)$. In vitro transmigration experiments have demonstrated that rings containing PLVAP and caveolin-1 surround lymphocytes during their migration (19). PLVAP was also reported to redistribute in the presence of and partially colocalize with vimentin and caveolin-1 (19). In capillary flow assays, tumor necrosis factor- $\alpha$ activated HUVECs grown in glass capillaries and incubated with anti-PLVAP antibody (19). As a result, the transmigration of peripheral blood mononuclear cells through the HUVEC layer was significantly inhibited, although the rolling and adhesion functions were unaffected (19). Blockage of PLVAP using a MECA-32 antibody in an acute peritonitis model decreased leukocyte migration by $\sim 85 \%$ (19). Therefore, PLVAP potentially aggravates inflammation by increasing leukocyte trafficking.

PLVAP controls the entry of lymphocytes into lymph nodes. Lymph nodes are specialized for efficient interaction of peripheral antigens with lymphocytes $(39,40)$. Caveolae, TECs 


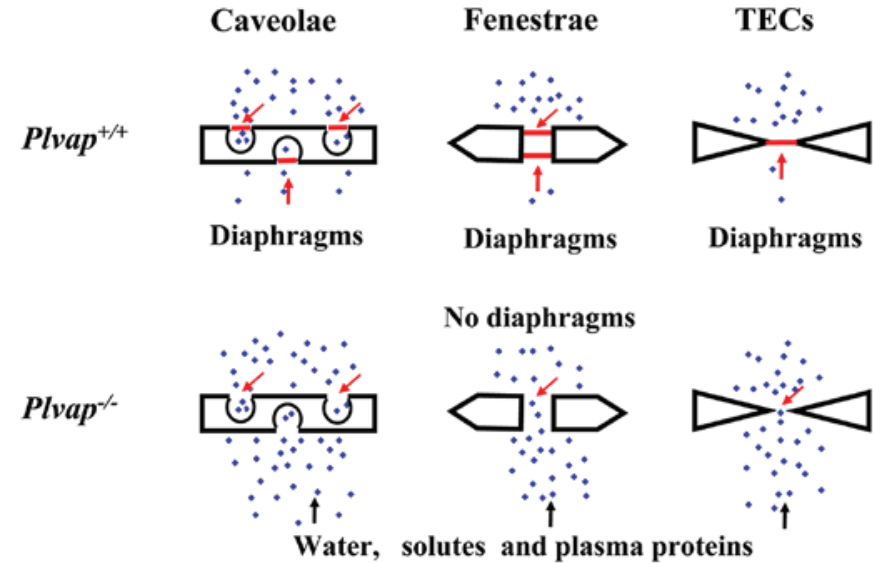

Figure 3. PLVAP and vascular permeability. PLVAP forms the stomatal and fenestral diaphragms that maintain the integrity of vasculature. Diaphragms are absent in $P L V A P^{-/}$mice, leading to the leakage of plasma proteins, water, solutes and small macromolecules. PLVAP, plasmalemma vesicle-associated protein; TECs, transendothelial channels.

and vesiculo-vacuolar organelles (VVOs) with diaphragms were identified in the subcapsular sinus lymphatic ECs (LECs) of lymph nodes (41). PLVAP is synthesized in LECs, which line the sinuses and cover the distal lymphatic vessels (41). Diaphragms consisting of PLVAP fibrils in TECs act as physical sieves that are responsible for regulating the entry of soluble antigens and lymphocytes into the parenchyma (41). PLVAP-null mice demonstrated an increase in lymphocyte transmigration through the sinus floor, in addition to non-selective antigen entry into the lymph system (41). Similarly, in the absence of PLVAP diaphragms on the sinusoidal floor LECs, the migration of lymph-generated lymphocytes to the parenchyma of peripheral lymph nodes (PLNs) is increased (41). When PLVAP-containing diaphragms interacted with antibodies, the entry of lymphocytes into the PLNs was prohibited at the sinus-cortex interface (41). These observations suggested that the selective entry of lymphocytes and antigens into the lymphatic nodes requires PLVAP (41).

In a previous study, migrating lymphocytes were demonstrated to produce F-actin-rich podosomes that probe the lymphatic sinus floor (41). The large PLVAP-positive patches observed on the LEC floor at points of transendothelial diapedesis in the sinus were colocalized with the areas populated by F-actin-rich podosomes (41). Electron microscopy revealed that, lymphocytes typically made contact with the wild-type LEC membrane in regions containing caveolae, VVOs, TECs and diaphragms (41). Therefore, PLVAP-positive diaphragms guarding the pores, tubular structures and channels may offer the path of least resistance through the sinusoidal floor LECs (41).

\section{PLVAP and diseases}

Cancer. With regard to cancerous tissues, PLVAP was first observed in malignant glioma microvasculature associated with increased fenestration, malperfusion and hyperpermeability (16). Subsequently, PLVAP was shown to be upregulated in ECs in tumors of the brain, lungs, breasts, stomach, liver, pancreas, colon, small intestine, kidneys, ovaries, prostate, uterus, skin and lymph nodes $(16,33,42)$. Furthermore, upregulation of PLVAP was reported to be similar in primary and metastatic tumors (18). Increased expression levels of PLVAP in tumors have been associated with angiogenesis (17). PLVAP is typically induced in large, well-vascularized tumors, in which PLVAP colocalizes with the vascular endothelial markers, cluster of differentiation 31 (CD31) and von Willebrand factor $(16,18,43)$. Furthermore, PLVAP was reported to be induced in vitro following exposure to VEGF-enriched medium and tumor cell lines (17).

As the presence of PLVAP facilitates vascular growth in cancer, it is considered a novel target for cancer therapy $(16,17)$. Downregulation of PLVAP using small hairpin RNA prevented the development of pancreatic adenocarcinoma in xenografts (32). In a previous study, infusion of recombinant monoclonal anti-PLVAP antigen-binding fragment co-expressed with the extracellular domain of human tissue factor into the feeding artery of hepatocellular carcinoma (HCC) led to vascular thrombosis and extensive necrosis of HCC (27). Furthermore, the suppression of tumor growth and minimal systemic toxicity indicated that PLVAP is a novel therapeutic target for the treatment of HCC (27).

Traumatic spinal cord injury. Spinal cord injury (SCI) is a potentially life-threatening condition $(44,45)$. Following SCI, both ECs and neurons are rapidly lost $(45,46)$. Expeditious and extensive cell death leads to inflammation followed by adaptive angiogenic responses at and around the epicenters of primary injury $(45,46)$. In contusive SCI mice, PLVAP was detected in several microvascular beds at $12 \mathrm{~h}$ post-SCI, with the level of PLVAP peaking at around 3-7 days post-injury (25). The majority of newly generated vessels at the injury sites were PLVAP-positive on day 3 post-injury, thus suggesting that PLVAP is expressed in neovasculature post-SCI (25). Furthermore, microvessels expressing PLVAP appeared to be spatially correlated with tissues containing actively extravasated neutrophils (25). On day 3 post-SCI, the expression of intracellular junction component zonula occludens-1 (ZO-1) and occludin was negligible in PLVAP-positive microvessels, which was indicative of a significant disruption of neurovascular integrity (25). Therefore, the upregulation of PLVAP may lead to secondary injury post-SCI via the induction of inflammation and deterioration of neurovascular function (25).

$T G$. Normal mature glomerular endothelium contains fenestrae without FDs for trafficking of macromolecules $(26,47)$ (Fig. 3). One of the histopathological features of TG is the upregulation of PLVAP expression and the increased numbers of caveolae in glomerular ECs (26). Transcytotic pathways that deliver albumin and immunoglobulins are largely dependent on vesicles originating from caveolae (20). As glomerular ECs in TG patients typically demonstrate reduced fenestration under the transmission electron microscope (26), Yamamoto et al (26) hypothesized that the elevation of PLVAP expression and caveolae formation may be a compensatory mechanism to increase the permeability of ECs to macromolecules. TG with double contouring of the glomerular capillaries is a characteristic manifestation of chronic rejection in an allograft recipient (48). The double contouring in TG is caused by endothelial injury and subsequent formation of a basement 
membrane-like structure beneath the endothelium; hence, the upregulation of PLVAP expression in glomerular capillaries may reflect vascular remodeling post-injury $(26,47,48)$.

Acute ischemic brain disease. In ischemic brain disease, damage to the blood-brain barrier results in an increased permeability of the microvasculature and fluid accumulation in the extracellular space, leading to brain ischemia, hypoxia and eventually mortality (43). PLVAP was suspected to be induced during hypoxia, as PLVAP upregulation was detected in the brain tissues of patients with acute ischemia (17). In C57/B6 mouse models with focal cerebral ischemia, negligible PLVAP staining was observed at $48 \mathrm{~h}$ following disease onset, and marked upregulation was detected 5 days later (43). Furthermore, all PLVAP-positive cells were shown to be located around the area of ischemic damage (43). Within the tissues, $\sim 17 \%$ of $C D 31$-positive vessels were expressing PLVAP by $48 \mathrm{~h}$ (43). As a result of PLVAP upregulation, no statistical difference was observed between the number of $P L V A P$-positive vessels and $C D 31$ expressing vessels on day 7 following disease onset (43).

Norrie disease. Norrie disease, which is characterized by abnormal angiogenesis and exudative vitreoretinopathy, is caused by mutations in the Norrie disease pseudoglioma $(N D P)$ gene (49-51). One-week-old NDP-null mice had reduced retinal capillarization (52), and increased endothelial fenestration and disrupted vascular integrity were observed in the retinas of $N D P$-null mice a week later (21). Unlike the wild-type mice, ectopic expression of PLVAP protein was demonstrated in the developing retinal vasculature of $N D P$-null mice, providing evidence for a potential role of PLVAP in the pathogenesis of Norrie disease (21).

Diabetic retinopathy $(D R)$. Loss of blood-retinal barrier (BRB) integrity is an important feature underlying the pathogenesis of diabetic macular edema (DME) (22). Increased retinal VEGF levels are associated with BRB breakdown in diabetic rodents, primates and humans $(22,23,34)$. VEGF is considered to induce transcellular transport via caveolae (22). In previous studies, exposure to exogenous VEGF led to an increase in the number of pinocytotic vesicles at the lumenal side of retinal capillaries, and to increased levels of PLVAP in caveolae $(23,53)$. To mimic the in vivo pathophysiology of DME, bovine retinal ECs (BRECs) were stimulated with VEGF, which led to an increase in the mRNA expression levels of caveolin-1 and PLVAP (22). In addition, a cell-based enzyme-linked immunosorbent assay detected a significant increase in the PLVAP content of BRECs at $72 \mathrm{~h}$ following VEGF treatment (23).

Mating the diabetic Ins2 $2^{\text {Akita }}$ (Akita) mice with the human VEGF photoreceptor-overexpressing trVEGF029 (Kimba) mice resulted in the Akimba (Ins $\left.2^{\text {Akita }} V E G F^{+/}\right)$mouse model that displayed retinal neovascularization and hyperglycemia, which are characteristics of advanced clinical DR (24). BRB loss, which was characterized by fluorescein leakage, was observed in both Kimba and Akimba mice (24). Among these mice, fluorescein leakage was associated with focal angiogenesis and PLVAP gene expression, suggesting a vital role for PLVAP in regulating the permeability of the BRB (24).

\section{Conclusion}

PLVAP is an endothelial cell-specific protein that is crucial for the development of SDs and FDs in subcellular structures, including caveolae, fenestrae and TECs (1-4). These diaphragms act as physical sieves that size-dependently control the exchange of soluble molecules between the blood plasma and interstitial fluid (10). In a previous study, PLVAP-deficient mice demonstrated increased premature mortality due to non-inflammatory protein severe enteropathy (10). Furthermore, PLVAP facilitates lymphocyte migration, presumably by sustaining the selective entry of lymphocytes into the parenchyma, in addition to offering the path of least resistance through cell bodies (39-41). Previous studies have also suggested that PLVAP is upregulated in various pathophysiological processes associated with angiogenesis, including tumorigenesis or the secondary injury of neurons following SCI $(16,25)$. In a previous study, PLVAP was shown to be a preferable therapeutic target for cancer therapy, since administration of PLVAP antibodies effectively suppressed tumor growth and had minimal systemic toxicity (27). Therefore, PLVAP may have a vital role in maintaining vascular integrity and homeostasis, under both normal and pathological conditions.

\section{Acknowledgements}

The present study was supported by a grant from the National Natural Science Foundation of China (grant no. 81370269). We are grateful for the support from the Shandong Taishan Scholarship, which was awarded to Dr Ju Liu.

\section{References}

1. Aird WC: Phenotypic heterogeneity of the endothelium: I. Structure, function and mechanisms. Circ Res 100: 158-173, 2007.

2. Aird WC: Phenotypic heterogeneity of the endothelium: II. Representative vascular beds. Circ Res 100: 174-190, 2007.

3. Tse D and Stan RV: Morphological heterogeneity of endothelium. Semin Thromb Hemost 36: 236-245, 2010.

4. Stan RV: Endothelial stomatal and fenestral diaphragms in normal vessels and angiogenesis. J Cell Mol Med 11: 621-643, 2007.

5. Stan RV, Ghitescu L, Jacobson BS and Palade GE: Isolation, cloning, and localization of rat PV-1, a novel endothelial caveolar protein. J Cell Biol 145: 1189-1198, 1999.

6. Stan RV: Structure of caveolae. Biochim Biophys Acta 1746: 334-348, 2005.

7. Bearer EL and Orci L: Endothelial fenestral diaphragms: A quick-freeze, deep-etch study. J Cell Biol 100: 418-428, 1985.

8. Simionescu M, Simionescu N, Silbert JE and Palade GE: Differentiated microdomains on the luminal surface of the capillary endothelium. II. Partial characterization of their anionic sites. J Cell Biol 90: 614-621, 1981.

9. Rostgaard J and Qvortrup K: Electron microscopic demonstrations of filamentous molecular sieve plugs in capillary fenestrae. Microvasc Res 53: 1-13, 1997.

10. Stan RV, Tse D, Deharvengt SJ, Smits NC, Xu Y, Luciano MR, McGarry CL, Buitendijk M, Nemani KV, Elgueta R, et al: The diaphragms of fenestrated endothelia: Gatekeepers of vascular permeability and blood composition. Dev Cell 23: 1203-1218, 2012.

11. Milici AJ, L'Hernault N and Palade GE: Surface densities of diaphragmed fenestrae and transendothelial channels in different murine capillary beds. Circ Res 56: 709-717, 1985.

12. Hallmann R, Mayer DN, Berg EL, Broermann R and Butcher EC: Novel mouse endothelial cell surface marker is suppressed during differentiation of the blood brain barrier. Dev Dyn 202: 325-332, 1995 . 
13. Niemela $H$, Elima $K$, Henttinen $T$, Irjala $H$, Salmi $M$ and Jalkanen S: Molecular identification of PAL-E, a widely used endothelial-cell marker. Blood 106: 3405-3409, 2005.

14. Stan RV, Tkachenko E and Niesman IR: PV1 is a key structural component for the formation of the stomatal and fenestral diaphragms. Mol Biol Cell 15: 3615-3630, 2004.

15. Ioannidou S, Deinhardt K, Miotla J, Bradley J, Cheung E, Samuelsson S, Ng YS and Shima DT: An in vitro assay reveals a role for the diaphragm protein PV-1 in endothelial fenestra morphogenesis. Proc Natl Acad Sci USA 103: 16770-16775, 2006.

16. Madden SL, Cook BP, Nacht M, Weber WD, Callahan MR, Jiang Y, Dufault MR, Zhang X, Zhang W, Walter-Yohrling J, et al: Vascular gene expression in nonneoplastic and malignant brain. Am J Pathol 165: 601-608, 2004.

17. Carson-Walter EB, Hampton J, Shue E, Geynisman DM, Pillai PK, Sathanoori R, Madden SL, Hamilton RL and Walter KA: Plasmalemmal vesicle associated protein-1 is a novel marker implicated in brain tumor angiogenesis. Clin Cancer Res 11: 7643-7650, 2005.

18. Liu Y, Carson-Walter EB, Cooper A, Winans BN, Johnson MD and Walter KA: Vascular gene expression patterns are conserved in primary and metastatic brain tumors. J Neurooncol 99: 13-24, 2010.

19. Keuschnigg J, Henttinen T, Auvinen K, Karikoski M, Salmi M and Jalkanen S: The prototype endothelial marker PAL-E is a leukocyte trafficking molecule. Blood 114: 478-484, 2009.

20. Minshall RD and Malik AB: Transport across the endothelium: Regulation of endothelial permeability. Handb Exp Pharmacol $107-144,2006$

21. Schafer NF, Luhmann UF, Feil S and Berger W: Differential gene expression in Ndph-knockout mice in retinal development. Invest Ophthalmol Vis Sci 50: 906-916, 2009.

22. Klaassen I, Hughes JM, Vogels IM, Schalkwijk CG, Van Noorden CJ and Schlingemann RO: Altered expression of genes related to blood-retina barrier disruption in streptozotocin-induced diabetes. Exp Eye Res 89: 4-15, 2009.

23. Wisniewska-Kruk J, Hoeben KA, Vogels IM, Gaillard PJ, Van Noorden CJ, Schlingemann RO and Klaassen I: A novel co-culture model of the blood-retinal barrier based on primary retinal endothelial cells, pericytes and astrocytes. Exp Eye Res 96: 181-190, 2012.

24. Wisniewska-Kruk J, Klaassen I, Vogels IM, Magno AL, Lai CM, Van Noorden CJ, Schlingemann RO and Rakoczy EP: Molecular analysis of blood-retinal barrier loss in the Akimba mouse, a model of advanced diabetic retinopathy. Exp Eye Res 122: 123-131, 2014

25. Mozer AB, Whittemore SR and Benton RL: Spinal microvascular expression of PV-1 is associated with inflammation, perivascular astrocyte loss, and diminished EC glucose transport potential in acute SCI. Curr Neurovasc Res 7: 238-250, 2010.

26. Yamamoto I, Horita $S$, Takahashi T, Tanabe K, Fuchinoue $S$, Teraoka S, Hattori M and Yamaguchi Y: Glomerular expression of plasmalemmal vesicle-associated protein-1 in patients with transplant glomerulopathy. Am J Transplant 7: 1954-1960, 2007.

27. Wang YH, Cheng TY, Chen TY, Chang KM, Chuang VP and Kao KJ: Plasmalemmal vesicle associated protein (PLVAP) as a therapeutic target for treatment of hepatocellular carcinoma. BMC Cancer 14: 815, 2014

28. Stan RV, Kubitza M and Palade GE: PV-1 is a component of the fenestral and stomatal diaphragms in fenestrated endothelia. Proc Natl Acad Sci USA 96: 13203-13207, 1999.

29. Hnasko R, McFarland M and Ben-Jonathan N: Distribution and characterization of plasmalemma vesicle protein-1 in rat endocrine glands. J Endocrinol 175: 649-661, 2002.

30. Stan RV, Arden KC and Palade GE: cDNA and protein sequence, genomic organization and analysis of cis regulatory elements of mouse and human PLVAP genes. Genomics 72: 304-313, 2001.

31. Stan RV: Multiple PV1 dimers reside in the same stomatal or fenestral diaphragm. Am J Physiol Heart Circ Physiol 286: H1347-H1353, 2004.

32. Deharvengt SJ, Tse D, Sideleva O, McGarry C, Gunn JR, Longnecker DS, Carriere C and Stan RV: PV1 down-regulation via shRNA inhibits the growth of pancreatic adenocarcinoma xenografts. J Cell Mol Med 16: 2690-2700, 2012.

33. Strickland LA, Jubb AM, Hongo JA, Zhong F, Burwick J, Fu L, Frantz GD and Koeppen H: Plasmalemmal vesicle-associated protein (PLVAP) is expressed by tumour endothelium and is upregulated by vascular endothelial growth factor-A (VEGF). J Pathol 206: 466-475, 2005.
34. Hofman P, Blaauwgeers HG, Vrensen GF and Schlingemann RO: Role of VEGF-A in endothelial phenotypic shift in human diabetic retinopathy and VEGF-A-induced retinopathy in monkeys. Ophthalmic Res 33: 156-162, 2001.

35. Hnasko R, Frank PG, Ben-Jonathan N and Lisanti MP: PV-1 is negatively regulated by VEGF in the lung of caveolin-1, but not caveolin-2, null mice. Cell Cycle 5: 2012-2020, 2006.

36. Herrnberger L, Seitz R, Kuespert S, Bösl MR, Fuchshofer R and Tamm ER: Lack of endothelial diaphragms in fenestrae and caveolae of mutant Plvap-deficient mice. Histochem Cell Biol 138: 709-724, 2012

37. Hisatsune H, Matsumura K, Ogawa M, Uemura A, Kondo N, Yamashita JK, Katsuta H, Nishikawa S, Chiba T and Nishikawa S: High level of endothelial cell-specific gene expression by a combination of the $5^{\prime}$ flanking region and the 5 ' half of the first intron of the VE-cadherin gene. Blood 105: 4657-4663, 2005.

38. Williamson JR and Grisham JW: Electron microscopy of leukocytic margination and emigration in acute inflammation in dog pancreas. Am J Pathol 39: 239-256, 1961

39. Girard JP, Moussion C and Förster R: HEVs, lymphatics and homeostatic immune cell trafficking in lymph nodes. Nat Rev Immunol 12: 762-773, 2012.

40. Germain RN, Robey EA and Cahalan MD: A decade of imaging cellular motility and interaction dynamics in the immune system. Science 336: 1676-1681, 2012

41. Rantakari P, Auvinen K, Jäppinen N, Kapraali M, Valtonen J, Karikoski M, Gerke H, Iftakhar-E-Khuda I, Keuschnigg J, Umemoto E, et al: The endothelial protein PLVAP in lymphatics controls the entry of lymphocytes and antigens into lymph nodes. Nat Immunol 16: 386-396, 2015.

42. Tichauer KM, Deharvengt SJ, Samkoe KS, Gunn JR, Bosenberg MW, Turk MJ, Hasan T, Stan RV and Pogue BW: Tumor endothelial marker imaging in melanomas using dual-tracer fluorescence molecular imaging. Mol Imaging Biol 16: 372-382, 2014.

43. Shue EH, Carson-Walter EB, Liu Y, Winans BN, Ali ZS, Chen J and Walter KA: Plasmalemmal vesicle associated protein-1 (PV-1) is a marker of blood-brain barrier disruption in rodent models. BMC Neurosci 9: 29, 2008.

44. Whetstone WD, Hsu JY, Eisenberg M, Werb $Z$ and Noble-Haeusslein LJ: Blood-spinal cord barrier after spinal cord injury: Relation to revascularization and wound healing. J Neurosci Res 74: 227-239, 2003.

45. Benton RL, Maddie MA, Minnillo DR, Hagg $T$ and Whittemore SR: Griffonia simplicifolia isolectin B4 identifies a specific subpopulation of angiogenic blood vessels following contusive spinal cord injury in the adult mouse. J Comp Neurol 507: 1031-1052, 2008

46. Casella GT, Bunge MB and Wood PM: Endothelial cell loss is not a major cause of neuronal and glial cell death following contusion injury of the spinal cord. Exp Neurol 202: 8-20, 2006.

47. Ichimura K, Stan RV, Kurihara H and Sakai T: Glomerular endothelial cells form diaphragms during development and pathologic conditions. J Am Soc Nephrol 19: 1463-1471, 2008.

48. Joosten SA, Sijpkens YW, van Kooten C and Paul LC: Chronic renal allograft rejection: Pathophysiologic considerations. Kidney Int 68: 1-13, 2005

49. Shastry BS, Hejtmancik JF and Trese MT: Identification of novel missense mutations in the Norrie disease gene associated with one X-linked and four sporadic cases of familial exudative vitreoretinopathy. Hum Mutat 9: 396-401, 1997.

50. Black GC, Perveen R, Bonshek R, Cahill M, Clayton-Smith J, Lloyd IC and McLeod D: Coats' disease of the retina (unilateral retinal telangiectasis) caused by somatic mutation in the NDP gene: A role for norrin in retinal angiogenesis. Hum Mol Genet 8: 2031-2035, 1999.

51. Chen ZY, Battinelli EM, Fielder A, Bundey S, Sims K, Breakefield XO and Craig IW: A mutation in the Norrie disease gene (NDP) associated with X-linked familial exudative vitreoretinopathy. Nat Genet 5: 180-183, 1993.

52. Luhmann UF, Lin J, Acar N, Lammel S, Feil S, Grimm C, Seeliger MW, Hammes HP and Berger W: Role of the Norrie disease pseudoglioma gene in sprouting angiogenesis during development of the retinal vasculature. Invest Ophthalmol Vis Sci 46: 3372-3382, 2005.

53. Hofman P, Blaauwgeers HG, Tolentino MJ, Adamis AP, Nunes Cardozo BJ, Vrensen GF and Schlingemann RO: VEGF-A induced hyperpermeability of blood-retinal barrier endothelium in vivo is predominantly associated with pinocytotic vesicular transport and not with formation of fenestrations. Vascular endothelial growth factor-A. Curr Eye Res 21: 637-645, 2000. 